\title{
HIST1H3D: A promising therapeutic target for lung cancer
}

\author{
YAN RUI $^{1 *}$, WEN-JIA PENG ${ }^{2 *}$, MING WANG $^{1 *}$, QIAN WANG $^{1 *}$, ZI-LI LIU $^{1}$, \\ YU-QING CHEN $^{1}$ and LI-NIAN HUANG ${ }^{1}$ \\ ${ }^{1}$ Department of Respiration and Critical Care Medicine, The First Affiliated Hospital of Bengbu Medical College,
Lung Cancer Diagnosis and Treatment Center of Anhui Province, Anhui Provincial Key Laboratory of
Clinical Basic Research on Respiratory Disease, Bengbu, Anhui 233004;
${ }^{2}$ Department of Epidemiology and Health Statistics, Bengbu Medical College, Bengbu, Anhui 233000, P.R. China
}

Received October 6, 2016; Accepted December 1, 2016

DOI: $10.3892 /$ ijo.2017.3856

\begin{abstract}
HIST1H3D gene encodes histone H3.1 and is involved in gene-silencing and heterochromatin formation. HIST1H3D expression is upregulated in primary gastric cancer tissue. In this study, we explored the effects of HIST1H3D expression on lung cancer, and its mechanisms. HIST1H3D expression was measured by immunohistochemistry and RT-PCR in lung cancer tissues and human lung cancer cell lines. Cell proliferation was assessed by MTT assay. Flow cytometric analysis was used to determine cell cycle distribution and apoptosis. Levels of related proteins were detected by western blotting. Bioinformatics analysis was performed to investigate related signaling pathways. cDNA microarray analysis was performed to identify differentially expressed genes following HIST1H3D knockdown. HIST1H3D expression was upregulated in lung cancer tissue samples and the H1299 human lung cancer cell line $(\mathrm{P}<0.01)$. Regulation of HIST1H3D expression in nucleus of cells in lung cancer tissues was significant associated with tumor stage $(\mathrm{P}=0.02)$ and lymph node metastases $(\mathrm{P}=0.04)$. Downregulation of HIST1H3D expression led to suppression of proliferation and colony forming ability, cell cycle arrest at the $\mathrm{G}_{0} / \mathrm{G}_{1}$ phase, and promotion of cell apoptosis. The microarray data revealed 522 genes that were differentially expressed after HIST1H3D knockdown in H1299 cells. These genes were shown to be linked to numerous pathways, including the cell cycle, p53 signaling, and MCM. Western blot analysis confirmed upregulated expression of the THBSI and TP53I3 genes, and downregulated expression of the CDK6,
\end{abstract}

Correspondence to: Dr Li-Nian Huang, Department of Respiration and Critical Care Medicine, The First Affiliated Hospital of Bengbu Medical College, Lung Cancer Diagnosis and Treatment Center of Anhui Province, Anhui Provincial Key Laboratory of Clinical Basic Research on Respiratory Disease, 287 Chang Huai Road, Bengbu, Anhui 233004, P.R. China

E-mail: bbmchln@126.com

*Contributed equally

Key words: lung cancer, HIST1H3D, H1299, colony formation, p53 signaling
CDKN1 and CCNE2 genes. In conclusion, our results suggest that HIST1H3D is highly expressed in lung cancer cell lines and tissues. Furthermore, HIST1H3D may be important in cell proliferation, apoptosis and cell cycle progression, and is implicated as a potential therapeutic target for lung cancer.

\section{Introduction}

Lung cancer is one of the most common cancers and the major cause of cancer deaths worldwide, with 1.6 million new lung cancer cases and 1.4 million lung cancer deaths each year (1). Although surgery is used to treat the early phase of lung cancer, the diagnosis of most patients is confirmed at an advanced stage when surgery is impossible (2). Chemotherapy and radiotherapy are used in clinical practice for lung cancer treatment, but the prognosis is poor, and the survival rate of patients with lung cancer is $<15 \%$ after 5 years (3). Therefore, it is critical to find novel strategies and effective methods for the treatment of lung cancer.

Histones are basic nuclear proteins that are involved in chromatin condensation in eukaryotic cells. There are five types of histones: H1/H5, H2A, H2B, H3 and H4 with no introns in their encoding genes, and no poly A tail in their transcripts (4-9). Increasing evidence has demonstrated that histones regulate chromatin states via covalent modification of amino acids, which further regulates gene expression. For instance, the less compacted chromatin domains show high transcriptional activity (10-12). The properties of histones in cancer cells are known to differ from those of noncancerous cells (13). A recent study demonstrated that histone mutations result in pediatric glioblastoma (14). Prostate cancer is associated with histone H2A.Z deregulation (15). HISTIH3D gene, located on chromosome 6, encodes histone H3.1, a member of the $\mathrm{H} 3$ class of histones in humans (16). H3.1 dimethylation of H3 lysine 9 is involved in gene-silencing and heterochromatin formation (17). Recently, it was shown that HIST1H3D expression is upregulated in primary gastric cancer tissue (18).

In this study, we investigated HIST1H3D expression in lung cancer tissues and human lung cancer cell lines, and explored the effects of HIST1H3D expression on lung cancer and signaling in proliferation and apoptosis. 


\section{Materials and methods}

Patients and tissue samples. Samples of lung cancer tissue and adjacent non-cancerous lung tissue were obtained from 74 patients hospitalized in the Department of Thoracic Surgery at the First Affiliated Hospital of Bengbu Medical College (Anhui, China) from July 2004 to September 2007. All subjects provided written informed consent. The experimental protocols were approved by the Ethics Committee of Bengbu Medical College. Clinicopathological features such as tumor size, pathological stage, lymph node metastasis and survival were recorded. Overall survival was calculated from the first surgical date to the date of death or the date of last follow-up (July 2012).

Cell culture and MTT cell proliferation assay. The H1299, H1688, H1975 and A549 human lung cancer cells were cultured in RPMI-1640 medium (Sigma, St. Louis, MO, USA) supplemented with $10 \%$ fetal bovine serum.

H1299 cells were plated in 96-well plates at a density of 2,000 cells/well. The cell viability was assessed on days 1,2 , $3,4$ and 5 . MTT ( $20 \mu \mathrm{l}, 5 \mathrm{mg} / \mathrm{ml})$ was added into each well. DMSO $(150 \mu \mathrm{l})$ was added to dissolve the crystals after $4 \mathrm{~h}$ of incubation. The optical density (OD) of each well was measured at $570 \mathrm{~nm}$ using an ELISA reader (Biotek ${ }^{\circledR}$ Elx800; Bio-Tek Instruments, Inc., Winooski, VT, USA).

Immunohistochemistry evaluation. HIST1H3D immunoreactivity in the nucleus was quantified based on a system in which scores of $0,1,2,3$ and 4 were assigned according to the percentage of positive cells at $0,1-25,26-50,51-75$ and $76-100 \%$, respectively (19). The staining intensity scores were defined as 0 (negative), $1(+), 2(++)$ and $3(+++)$. The product of the positive cells and intensity score was calculated. High and low expression of HIST1H3D in the nucleus was defined as $>1.5$ and $\leq 1.5$, respectively.

Lentiviral construction of small-interfering RNA (siRNA) treatment. HIST1H3D-specific siRNA (5'-GCT GAT TCG CAA ACT GCC ATT-3') and the negative control (NC) sequence (5'-TTC TCC GAA CGT GTC ACG T-3') were cloned into the AgeI and EcoRI sites of the pGCSIL-GFP lentiviral vector (GeneChem, Shanghai, China) to generate the pGCSIL-GFP-HIST1H3D-siRNA plasmid for specific inhibition of HISTIH3D expression and the pGCSIL-GFP-NC plasmid as the NC.

Inhibition of HISTIH3D expression in H1299 cells with HIST1H3D-specific siRNA was performed. H1299 cells were seeded into 6 -well plates $\left(5 \times 10^{4}\right.$ cells/well). NC siRNA (5'-TTC TCC GAA CGT GTC ACG T-3') and HIST1H3D-specific siRNA (5'-GCT GAT TCG CAA ACT GCC ATT-3') were transfected into the cells using a viral system at a multiplicity of infection (MOI) according to the manufacturer's protocol.

Quantitative real-time PCR ( $q R T-P C R)$. Total RNA was extracted from H1299 cells using TRIzol reagent and cDNA was generated from a sample of total RNA $(2 \mu \mathrm{g})$ by reverse transcription using the PrimeScript ${ }^{\circledR}$ RT kit. Total RNA was used for reverse transcription. RT-PCR was performed with the SYBR ${ }^{\circledast}$ PrimeScript ${ }^{\mathrm{TM}}$ RT-PCR kit (Takara, Kyoto, Japan) according to the manufacturer's instructions. The specific sequences of primers were as follows: HIST1H3D (forward, 5'-TTC GCA AAC TGC CAT TCC-3' and reverse, 5'-GAG CCT TTG GGT TTT GGT T-3'), and GAPDH (forward, 5'-TGA CTT CAA CAG CGA CAC CCA-3' and 5'-CAC CCT GTT GCT GTA GCC AAA-3'). GAPDH was used as an internal control. Primers were designed by Beacon Designer 2 software. The $2^{-\Delta \Delta C \mathrm{CT}}$ method was used to analyze the relative changes in gene expression.

Western blot analysis. Lung cancer tissue and adjacent non-cancerous tissues or H1299 cells were harvested and homogenized in a pre-chilled lysis buffer (Sangon Biotech). Homogenates were centrifuged at $12,000 \mathrm{x} \mathrm{g}$ for $20 \mathrm{~min}$ at $4^{\circ} \mathrm{C}$. Equal amounts of denatured proteins were separated by SDS-PAGE and then transferred to PVDF membranes. To block non-specific binding, membranes were incubated in TBST containing 5\% fat-free milk for $2 \mathrm{~h}$. The membranes were then incubated with primary antibodies (polyclonal anti-HIST1H3D, anti-CCNE2, anti-CDK6, anti-CDKN1A, anti-THBS1, anti-TP53I3 and anti-GAPDH, 1:1,000 dilution) overnight at $4^{\circ} \mathrm{C}$. After washing with TBST, membranes were incubated with an HRP-conjugated secondary antibody (1:1,000 dilution; Sangon Biotech) for $2 \mathrm{~h}$ at room temperature. After being rinsed with washing buffer, immunoreactive bands were visualized using EasyBlot ECL kit (Sangon Biotech).

Cell growthassay. Cell growth was determined using a Cellomics ArrayScan (GeneChem). Infected cells (800 cells/well) were seeded into 96-well plates and cell growths was assayed at 1,2, 3, 4 and 5 days using a Cellomics ArrayScan (Cellomics Inc., Pittsburgh, PA, USA). Cell growth curves were constructed for each cell type.

Flow cytometric analyses. For cell cycle analysis, cells $(2,000$ cells/well $)$ were cultured in 6 -well plates. At 3 days post-infection with HIST1H3D-siRNA lentivirus or control lentivirus, cells were then collected, washed twice with icecold PBS and fixed with $70 \%$ ethanol at $4^{\circ} \mathrm{C}$. The cells were then stained with PBS containing $50 \mu \mathrm{g} / \mathrm{ml}$ propidium iodide (PI, $2 \mathrm{mg} / \mathrm{ml}$; Sigma-Aldrich) in the presence of $100 \mu \mathrm{g} / \mathrm{ml}$ of RNase (10 mg/ml; Sangon Biotech). For analysis of cell apoptosis, the cells were stained with $100 \mu 1$ binding buffer containing $5 \mu 1$ Annexin V-APC (BD Bioscience, San Diego, CA, USA) at room temperature in the dark for 10-15 min. Cells were analyzed by flow cytometry using FACSCalibur (BD Biosciences).

Colony formation assay. H1299 cells were plated in 6-well plates (500 cells/well) and cultured at $37^{\circ} \mathrm{C}$ in a humidified atmosphere containing $5 \% \mathrm{CO}_{2}$ for 14 days to allow colony formation. At the indicated time-points, cells were washed twice with PBS, treated with Giemsa stain for $20 \mathrm{~min}$, and then washed several times with $\mathrm{ddH}_{2} \mathrm{O}$. The number of colonies was counted under a fluorescence microscope (MicroPublisher 3.3RTV; Olympus, Tokyo, Japan).

Gene expression profiling. After transfection, total RNA was extracted from H1299 cells using TRIzol reagent. RNA quality was assessed by NanoDrop 2000 and Agilent 

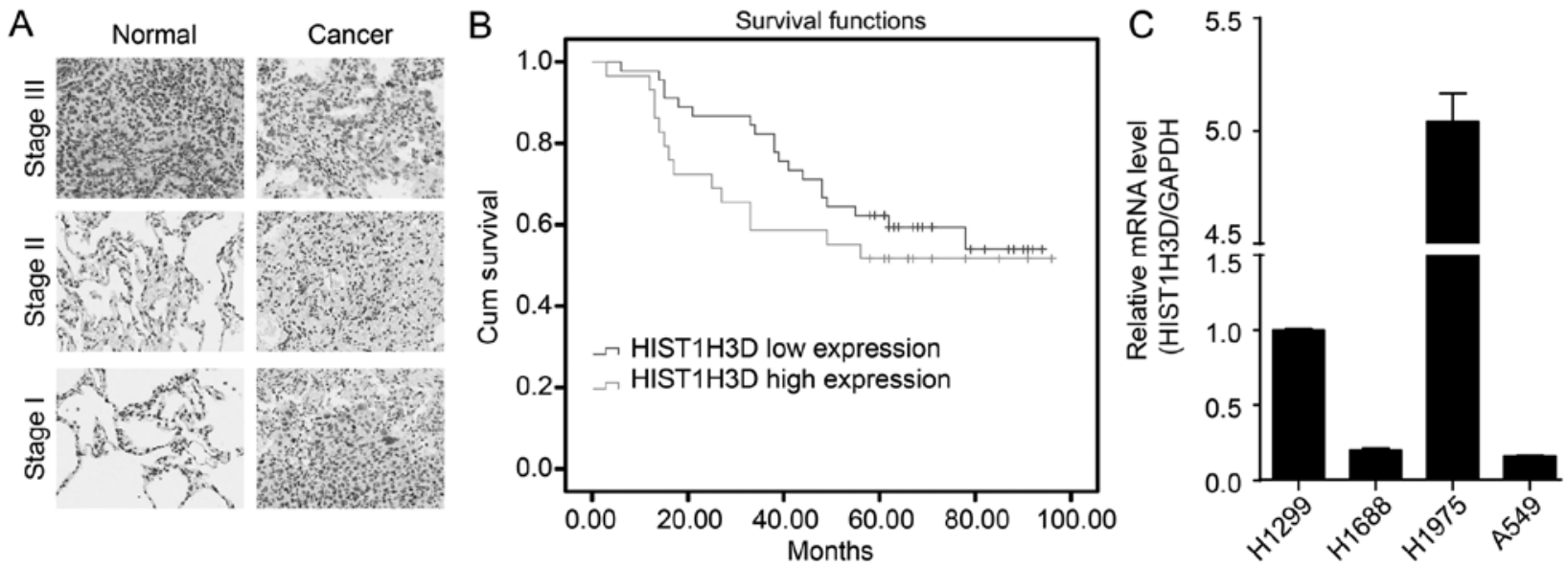

Figure 1. HIST1H3D expression in lung cancer tissue samples and four cell lines. (A) Immunohistochemical staining showing that HIST1H3D is expressed at higher levels in lung cancer tissue than in adjacent non-cancerous tissue. (B) Kaplan-Meier survival curve showing the correlation of overall survival with high and low expression of HIST1H3D in lung cancer $(\mathrm{P}=0.35)$. (C) qRT-PCR analysis of the expression of HIST1H3D mRNA in four cell lines. GAPDH gene was used as an internal control.

Table I. Relationship between HIST1H3D expression and clinicopathological characteristics of patients with lung cancer.

\begin{tabular}{|c|c|c|c|}
\hline \multirow[b]{2}{*}{ Variable } & \multicolumn{2}{|c|}{ HIST1H3D expression } & \multirow[b]{2}{*}{ P-value } \\
\hline & Low & High & \\
\hline \multicolumn{4}{|l|}{ Gender } \\
\hline Male & 24 & 15 & 0.89 \\
\hline Female & 21 & 14 & \\
\hline \multicolumn{4}{|l|}{ Age (years) } \\
\hline$\leq 60$ & 25 & 16 & 0.89 \\
\hline$>60$ & 20 & 13 & \\
\hline \multicolumn{4}{|c|}{ Tumor size (cm) } \\
\hline$\leq 3.5$ & 25 & 13 & 0.37 \\
\hline$>3.5$ & 20 & 16 & \\
\hline \multicolumn{4}{|l|}{ Stage } \\
\hline I & 11 & 0 & 0.02 \\
\hline II & 22 & 19 & \\
\hline III & 12 & 10 & \\
\hline \multicolumn{4}{|c|}{ Lymph node metastasis } \\
\hline $\mathrm{Nx} / \mathrm{NO}$ & 35 & 16 & $0.04^{\mathrm{a}}$ \\
\hline $\mathrm{N} 1 / \mathrm{N} 2 / \mathrm{N} 3$ & 10 & 13 & \\
\hline \multicolumn{4}{|c|}{ Distant metastasis } \\
\hline M0 & 43 & 28 & 0.81 \\
\hline M1 & 2 & 1 & \\
\hline
\end{tabular}

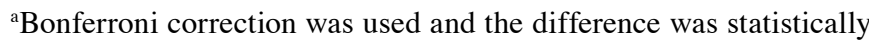
significant.

Bioanalyzer 2100. Gene expression profiling was performed using human gene arrays on the Affymetrix GeneChip PrimeView platform according to the manufacturer's protocol. Differentially expressed genes were identified on the basis of a 1.5 -fold change in expression with P-values $<0.05$. KEGG and
BioCarta pathway analyses were performed to investigate the functional and pathway annotation of all the selected genes.

Statistical analyses. Data were expressed as mean \pm standard deviation (SD). The difference between two groups was determined by Student's t-test or Wilcoxon's rank test. Correlations between HIST1H3D expression and clinicopathological characteristics were determined using Pearson's $\chi^{2}$ test. Survival analysis was performed using the log-rank test. All statistical analyses were performed using SPSS 12.0 software. A value of $\mathrm{P}<0.05$ was considered to indicate statistical significance.

\section{Results}

HISTIH3D expression in lung cancer cells and tissues. Our investigation of the expression of HIST1H3D mRNA in lung cancer and the adjacent non-cancerous tissues showed that the level of HIST1H3D protein in lung cancer tissues was significantly increased, compared with the adjacent non-cancerous tissues $(\mathrm{P}<0.01)$ (Fig. 1A). Kaplan-Meier survival analysis suggested that HIST1H3D expression in lung cancer tissues was not associated with that in the adjacent non-cancerous tissues (P-value for log-rank, 0.35) (Fig. 1B). Furthermore, we determined that HIST1H3D mRNA was highly expressed in the lung cancer cell lines H1299 and H1975 (Fig. 1C). However, HIST1H3D mRNA was expressed at low levels in the lung cancer cell lines A459 and H1688. The discrepancy of HIST1H3D expression in different lung cancer cell lines may be due to tumor heterogeneity.

In addition, higher levels of HIST1H3D were observed in lung cancer tissues at stage III compared with the levels observed at stage I/II $(\mathrm{P}<0.05)$ (Table I), with higher expression in the cases with lymph node metastasis compared to those without $(\mathrm{P}<0.05)$ (Table I). However, there was no significant difference in HIST1H3D expression between the genders, nor a significant association with age, tumor size, and distant metastasis $(\mathrm{P}>0.05)$ (Table I).

Lentiviral transfection with HISTIH3D suppresses HISTIH3D expression. HIST1H3D siRNA or control lentivirus was 


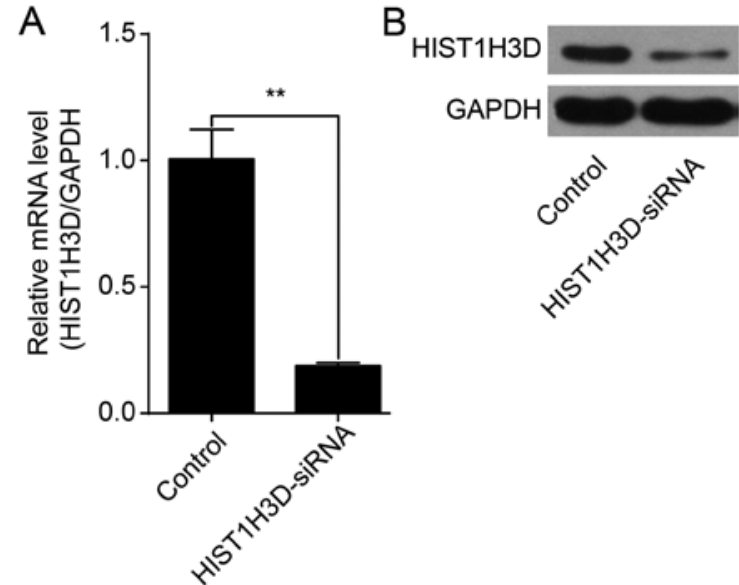

Figure 2. Small-interfering RNA-mediated inhibition of HIST1H3D expression following lentiviral transfection. (A) qRT-PCR analysis of HIST1H3D mRNA levels. (B) Western blot analysis of HIST1H3D protein levels (GAPDH was used as a loading control).

transfected into H1299 cells. HIST1H3D mRNA levels were significantly reduced by $81 \%$ in cells transfected with HIST1H3D siRNA compared to cells transfected with NC siRNA $(\mathrm{P}<0.01)$ (Fig. 2A). Western blot analysis showed that HIST1H3D protein expression was decreased in cells transfected with HIST1H3D siRNA (Fig. 2B).
Effect of siRNA-mediated knockdown of HIST1H3D on cell proliferation. To evaluate the effect of HIST1H3D on cell proliferation, we determined proliferation of H1299 cells transfected with HIST1H3D-specific siRNA. The number of cells in the control group increased in a time-dependent manner, while only a slight increase in cell numbers was observed in the HIST1H3D-siRNA group (Fig. 3A and B), with a significant difference in cell numbers between the Control and HIST1H3D-siRNA groups observed at 5 days post-infection $(\mathrm{P}<0.01)$ (Fig. 3B).

H1299 cell proliferation was also evaluated by MTT assay. The results showed that HIST1H3D-siRNA group cell growth at day 5 were significantly inhibited compared to control group $(\mathrm{P}<0.01)$ (Fig. 3C).

Effect of HIST1H3D siRNA on cell colony formation. As shown in Fig. 4, the number of colonies was decreased by $64.6 \%$ in H1299 cells transfected with HIST1H3D siRNA compared with those transfected with NC siRNA $(\mathrm{P}<0.01)$.

Effect of HISTIH3D siRNA on cell apoptosis. To determine the effect of HIST1H3D expression on cell survival, cell apoptosis was analyzed by a flow cytometry. As shown in Fig. 5, the number of apoptotic cells transfected with HIST1H3D siRNA was significantly greater than that when compared with the number of NC siRNA transfected cells $(\mathrm{P}<0.01)$.

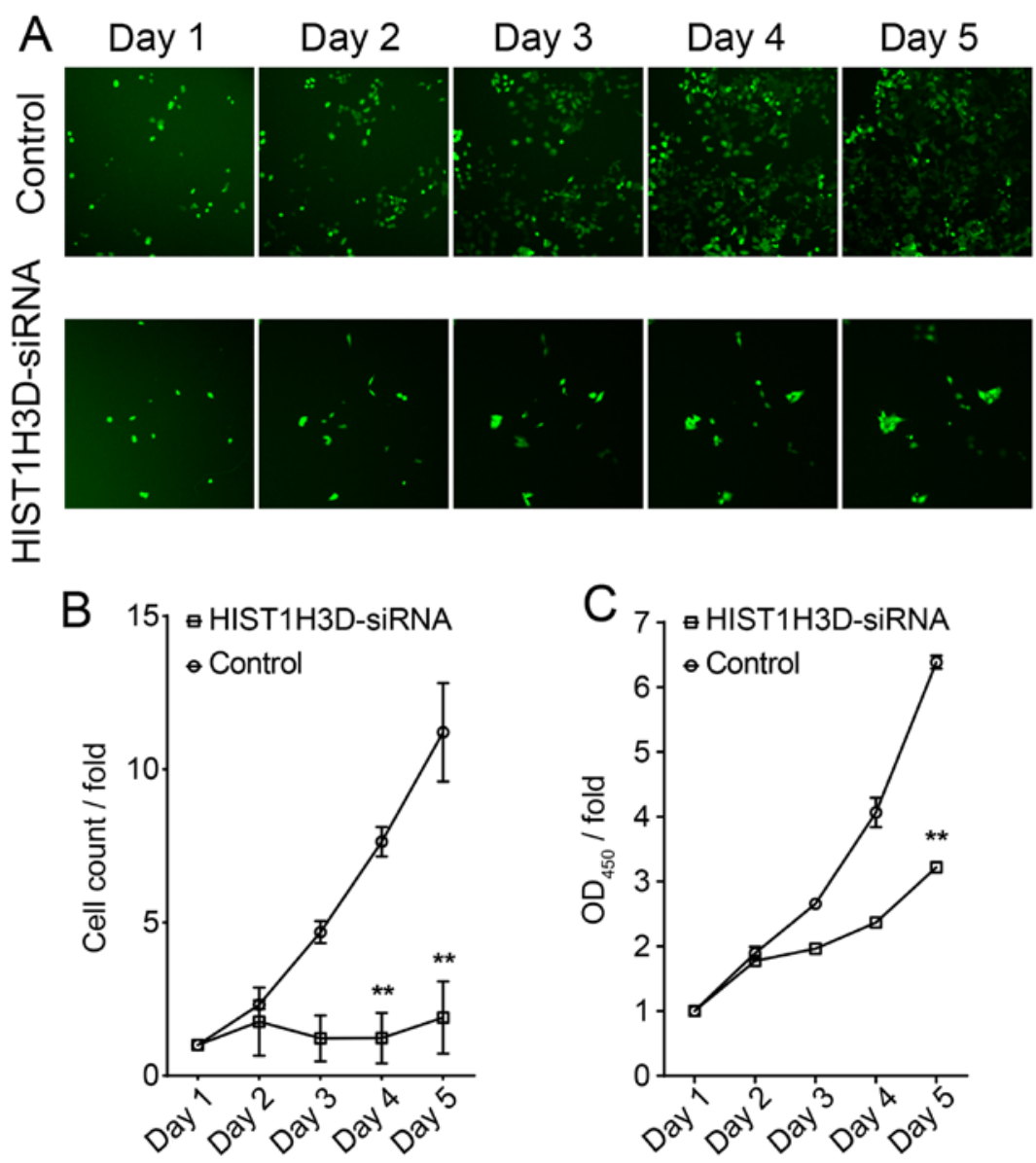

Figure 3. HIST1H3D knockdown inhibits H1299 cell growth. (A) Representative fluorescence microscopy image taken at daily intervals after lentiviral infection. (B) Daily post-infection cell counts measured using an automated cell counter (control vs. HIST1H3D-siRNA at day 5, ${ }^{* *} \mathrm{P}<0.01$ ). (C) Post-infection cell proliferation measured by MTT assay (control vs. HIST1H3D-siRNA at day $5,{ }^{* *} \mathrm{P}<0.01$ ). 

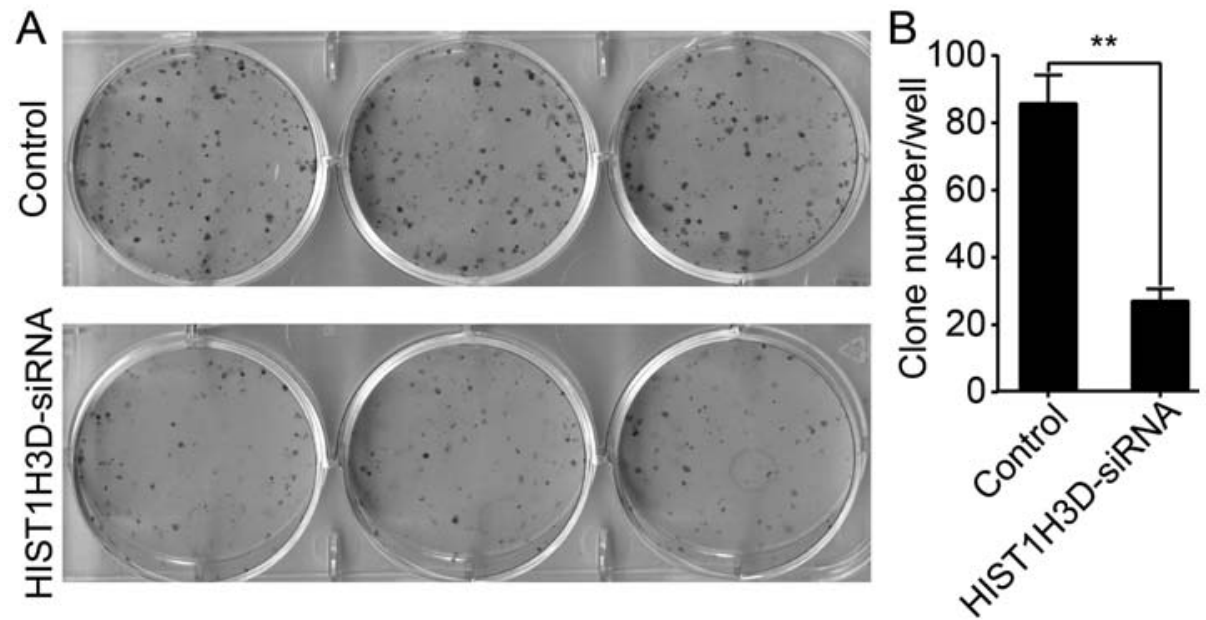

Figure 4. HIST1H3D knockdown inhibits H1299 cell colony formation. (A) Photomicrographs of Giemsa-stained H1299 colonies in 6-well plates. (B) Mean number of cells in each colony/well, ${ }^{* *} \mathrm{P}<0.01$.
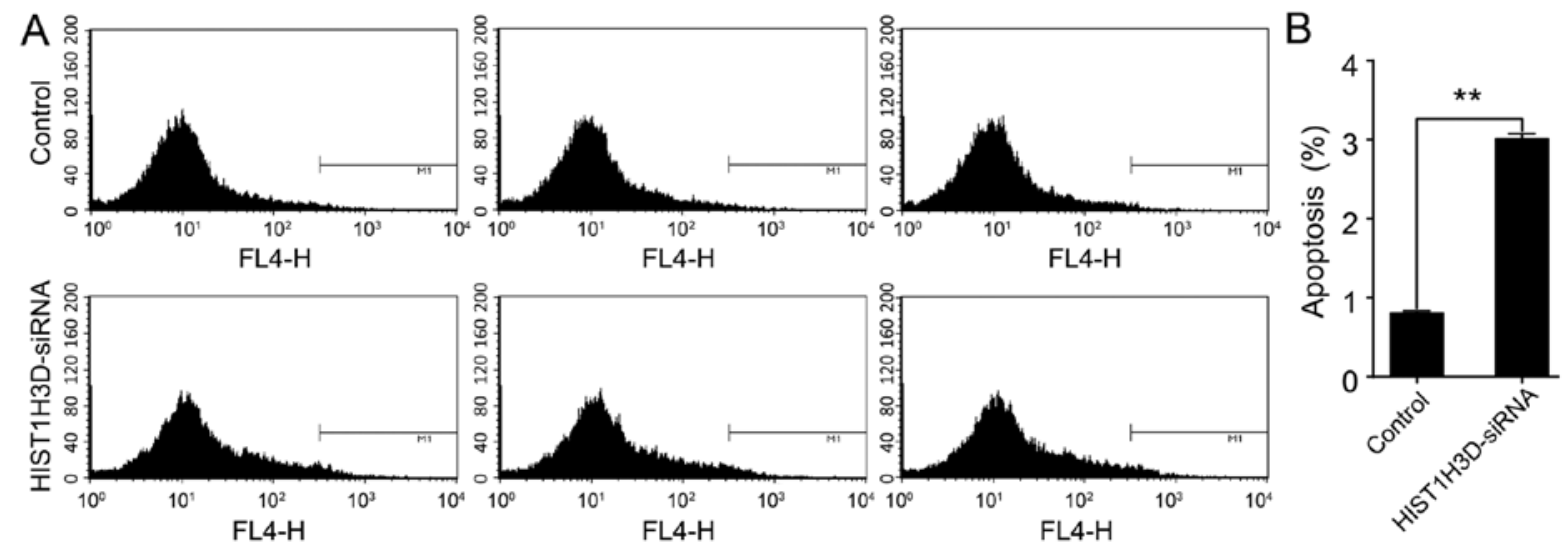

Figure 5. HISTIH3D knockdown increased H1299 cell apoptosis. (A) Graphs showing apoptosis in each sample (in triplicate) determined by Annexin V staining. (B) Percentage of apoptotic cells calculated on the basis of the results shown in (A). ${ }^{* *} \mathrm{P}<0.01$.

Effect of HISTIH3D siRNA on cell cycle distribution. Cell cycle analysis showed that, following transfection with HIST1H3D siRNA, 67.9, 26.2 and $5.9 \%$ of the cells were in the $\mathrm{G}_{0} / \mathrm{G}_{1}, \mathrm{~S}$ and $\mathrm{G}_{2} / \mathrm{M}$ phases, respectively (Fig. 6). Following transfection with NC siRNA, 38.2, 55.9 and 5.9\% of the cells were in the $\mathrm{G}_{0} / \mathrm{G}_{1}, \mathrm{~S}$ and $\mathrm{G}_{2} / \mathrm{M}$ phases, respectively. The result suggested that inhibition of HIST1H3D-siRNA arrested cells in the $\mathrm{G}_{0} / \mathrm{G}_{1}$ phase $(\mathrm{P}<0.01)$.

Profiling of expression of genes regulated by HISTIH3D. To determine the effects of HIST1H3D on the potential pathways involved in the development of lung cancer, we performed microarray analysis to identify the differential gene expression profiles between cells following siRNA-mediated knockdown of HIST1H3D. A total of 522 differentially expressed genes (284 upregulated and 238 downregulated) were identified following HIST1H3D knockdown (Fig. 7A). KEGG and BioCarta pathway analysis showed that HIST1H3D-regulated genes were involved mainly in the cell cycle progression, p53 signaling, and MCM (Fig. 7B).

Most of the DNA microarray results were validated by western blot assays (Fig. 7C). We observed upregulated expression of the THBSI and TP53I3 genes, and downregulated expression of the CDK6, CDKN1 and CCNE2 genes in si-HIST1H3D group when compared to the control group (Fig. 7D).

\section{Discussion}

Histones are basic nuclear proteins that are involved in regulation of chromatin structure in eukaryotic cells. Histones undergo post-translational modifications of the their N-terminal tail, such as methylation, acetylation, phosphorylation, citrullination, SUMOylation, ubiquitination and ADP-ribosylation (12). These modifications alter chromatin structure, with a critical role in regulating gene expression. Abnormal histone modifications can lead to changes in the chromosome structure and gene transcription, and also plays a critical role in cancer cell proliferation, differentiation and apoptosis $(20,21)$.

Accumulating evidence shows that histones contribute to the pathogenesis of various malignancies $(22,23)$. Previous studies have shown that histone $\mathrm{H} 3 \mathrm{mRNA}$ is accumulated in tumors, and upregulation of histone $\mathrm{H} 3 \mathrm{mRNA}$ expression increases the proliferative activity of tumor cells $(24,25)$. Furthermore, recent studies suggest that histone cluster genes are overexpressed in 

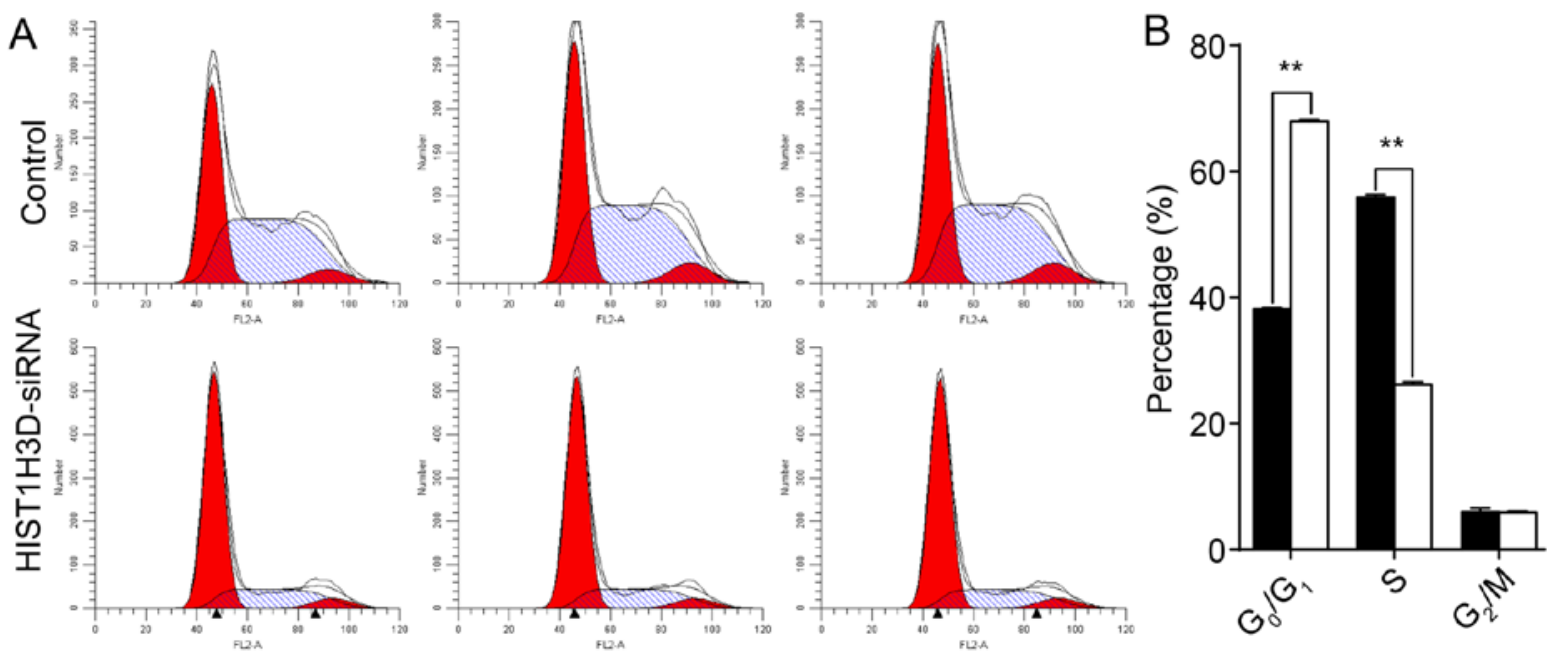

Figure 6. HISTIH3D knockdown leads to cell cycle arrest. (A) Cell cycle phase distribution following transfection with HIST1H3D siRNA and NC siRNA (triplicate samples for each treatment). (B) Cell cycle phase distribution expressed as a percentage of total cells, ${ }^{* *} \mathrm{P}<0.01$.

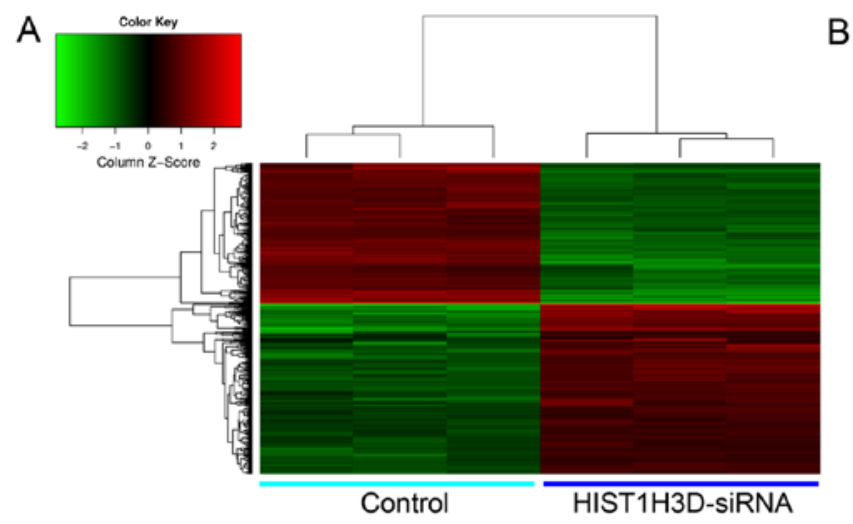

B
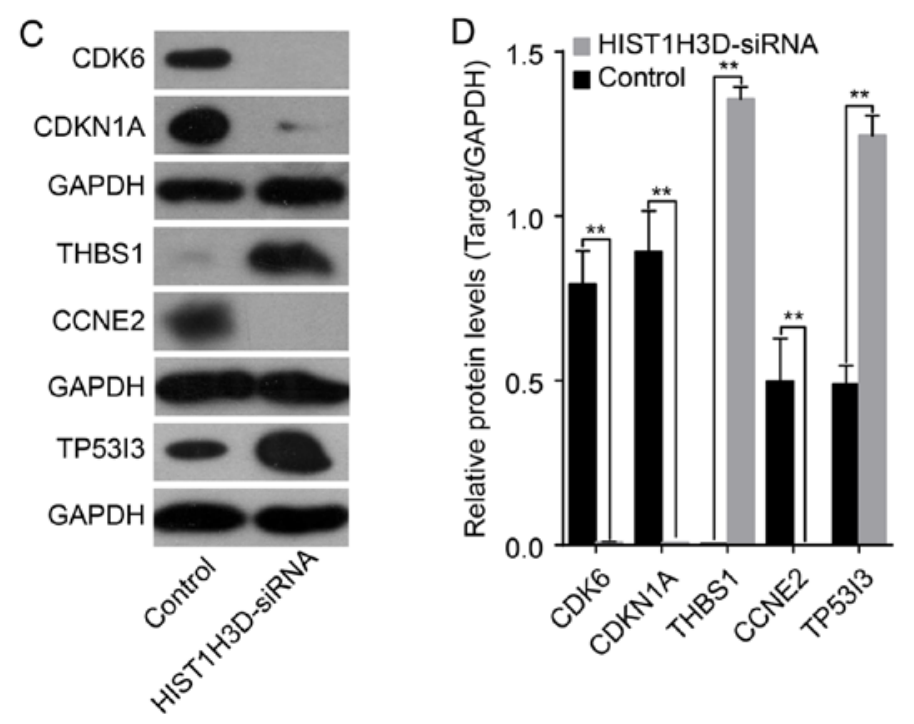

Figure 7. Knockdown of HIST1H3D in H1299 cells significantly alters gene expression. (A) Hierarchical clustering of differentially expressed transcripts. (B) KEGG and BioCarta pathway enrichment of differentially expressed genes in H1299 cells transfected with HIST1H3D lentivirus compared with the control. (C) Western blotting and (D) densitometric analyses of target proteins in the p53 signaling pathway in H1299 cells transfected with control lentivirus and HIST1H3D lentivirus. Data represent the mean \pm SD of arbitrary densitometric values of targets/GAPDH. "P<0.01.

several types of malignancies. Sadikovic et al performed integrative whole-genome analysis of DNA copy number, promoter methylation and gene expression in 10 osteosarcomas, and identified overexpression of histone cluster 2 genes at chromosome 1q21.1-q21.3 (26). In microarray-based expression profiling of meningiomas, and Pérez-Magán et al identified overexpression of 16 histone cluster 1 genes located on chromosome $6 p$ in recurrent meningiomas (27). 
In the present study, we first determined the HIST1H3D expression in lung cancer tissues and adjacent non-cancerous tissues. Results indicated that HIST1H3D expression was increased in lung cancer tissues compared with that in adjacent non-cancerous tissues. We observed higher expression of HIST1H3D in patients with advanced disease (TNM II/III) and lymph node metastasis (N1/N2/N3), while there were no significant differences in HIST1H3D expression between the genders. Furthermore, there were no significant associations of HIST1H3D expression with age, tumor size or distant metastasis. Furthermore, we demonstrated increased HIST1H3D expression in two lung cancer cell lines H1299 and H1975 and decreased HIST1H3D expression in A459 and H1688 cells. These findings suggest that HIST1H3D expression may be associated with tumor heterogeneity in lung cancer. Iwaya et al found that HIST1H3D is associated with the development of high-risk lung cancer (18). Thus, association between HIST1H3D expression and the risk of lung cancer requires further investigation.

To assess the effects of HIST1H3D expression on lung cancer, we reduced the HIST1H3D expression by RNA interference (RNAi) in H1299 human lung cancer cells. RNAi is a powerful genetic tool for silencing gene expression through degradation of specific mRNA (28-31). After confirming siRNA-mediated knockdown of HIST1H3D in H1299 cells, we observed reduced proliferation and colony formation as well as increased apoptosis. These results suggest that upregulation of HIST1H3D expression promotes tumor cell development. However, the mechanisms by which HIST1H3D knockdown inhibits proliferation and increases apoptosis remain to be clarified due to the discrepancy between suppression of cell proliferation and increase of apoptosis ratio.

The cell cycle is the series of events that lead to cell division. We performed flow cytometric cell cycle analysis to elucidate the mechanism underlying the effects of HIST1H3D on the control of cell growth. Interestingly, we found that HIST1H3D knockdown induced $G_{0} / G_{1}$ cell cycle arrest, indicating that HIST1H3D promotes human lung cancer cell proliferation and colony formation by modulating cell cycle progression. However, the mechanism responsible for cell cycle arrest of HIST1H3Dsilenced cells remains to be clarified in future studies.

The tumor suppressor p53 plays key roles in regulating the cell cycle and apoptosis by modulating the expression of target genes $(32,33)$. In this study, cDNA microarray analysis indicated that the 553 signaling pathway is triggered following HIST1H3D-knockdown, these results were validated by western blot analysis. Differential expression of a number of genes in the p53 signaling pathway was detected, with upregulation of genes such as THBSI and TP53I3 and downregulation of other genes, including CDK6, CDKN1 and CCNE2. These results suggest that the HIST1H3D is involved in the development of lung cancer through regulating the expression of these genes. Although the current data are too limited to support an in-depth hypothesis, our results provide a direction for future research.

In conclusion, our results show that HIST1H3D expression is upregulated in lung cancer tissues and cell lines. Furthermore, this study demonstrates that siRNA-mediated downregulation of HIST1H3D expression inhibits cell growth, proliferation and colony formation, while promoting cell apoptosis, and cycle arrest in the $\mathrm{G}_{0} / \mathrm{G}_{1}$ phase. These findings indicate that HIST1H3D plays an important role in lung cancer development and implicate HIST1H3D as a therapeutic target in lung cancer.

\section{Acknowledgements}

This study was supported by the Personnel training funds of Anhui Province Health and Family Planning Commission, the Natural Science Fund of Education Department of Anhui Province (KJ2015B070), and the Doctor's Scientific Research Foundation of Bengbu Medical College.

\section{References}

1. Torre LA, Bray F, Siegel RL, Ferlay J, Lortet-Tieulent J and Jemal A: Global cancer statistics, 2012. CA Cancer J Clin 65: 87-108, 2015

2. Chen W, Li Z, Bai L and Lin Y: NF-kappaB in lung cancer, a carcinogenesis mediator and a prevention and therapy target. Front Biosci (Landmark Ed) 16: 1172-1185, 2011.

3. Vischioni B, Oudejans JJ, Vos W, Rodriguez JA and Giaccone G: Frequent overexpression of aurora B kinase, a novel drug target, in non-small cell lung carcinoma patients. Mol Cancer Ther 5: 2905-2913, 2006.

4. Birnstiel ML, Busslinger M and Strub K: Transcription termination and 3' processing: The end is in site! Cell 41: 349-359, 1985.

5. Liu TJ, Levine BJ, Skoultchi AI and Marzluff WF: The efficiency of 3'-end formation contributes to the relative levels of different histone mRNAs. Mol Cell Biol 9: 3499-3508, 1989.

6. Osley MA: The regulation of histone synthesis in the cell cycle. Annu Rev Biochem 60: 827-861, 1991

7. Zanier K, Luyten I, Crombie C, Muller B, Schümperli D, Linge JP, Nilges M and Sattler M: Structure of the histone mRNA hairpin required for cell cycle regulation of histone gene expression. RNA 8: 29-46, 2002.

8. Meshi T, Taoka KI and Iwabuchi M: Regulation of histone gene expression during the cell cycle. Plant Mol Biol 43: 643-657, 2000.

9. Schümperli D: Cell-cycle regulation of histone gene expression. Cell 45: 471-472, 1986.

10. Turner BM: Cellular memory and the histone code. Cell 111: 285-291, 2002.

11. Berger SL: Histone modifications in transcriptional regulation. Curr Opin Genet Dev 12: 142-148, 2002.

12. Jenuwein $\mathrm{T}$ and Allis CD: Translating the histone code. Science 293: 1074-1080, 2001.

13. Cruft HJ, Mauritzen CM and Stedman E: Abnormal properties of histones from malignant cells. Nature 174: 580-585, 1954.

14. Rheinbay E, Louis DN, Bernstein BE and Suvà ML: A tell-tail sign of chromatin: Histone mutations drive pediatric glioblastoma. Cancer Cell 21: 329-331, 2012.

15. Dryhurst D and Ausió J: Histone H2A.Z deregulation in prostate cancer. Cause or effect? Cancer Metastasis Rev 33: 429-439, 2014.

16. Albig W, Kioschis P, Poustka A, Meergans K and Doenecke D: Human histone gene organization: Nonregular arrangement within a large cluster. Genomics 40: 314-322, 1997.

17. Peters AH and Schübeler D: Methylation of histones: Playing memory with DNA. Curr Opin Cell Biol 17: 230-238, 2005.

18. Iwaya T, Fukagawa T, Suzuki Y, Takahashi Y, Sawada G, Ishibashi M, Kurashige J, Sudo T, Tanaka F, Shibata K, et al: Contrasting expression patterns of histone mRNA and microRNA 760 in patients with gastric cancer. Clin Cancer Res 19: 6438-6449, 2013.

19. Wang B, Tang J, Liao D, Wang G, Zhang M, Sang Y, Cao J, Wu Y, Zhang $\mathrm{R}, \mathrm{Li} \mathrm{S}$, et al: Chromobox homolog 4 is correlated with prognosis and tumor cell growth in hepatocellular carcinoma. Ann Surg Oncol 20 (Suppl 3): S684-S692, 2013.

20. Esteller M: Cancer epigenomics: DNA methylomes and histonemodification maps. Nat Rev Genet 8: 286-298, 2007.

21. Berdasco $\mathbf{M}$ and Esteller M: Aberrant epigenetic landscape in cancer: How cellular identity goes awry. Dev Cell 19: 698-711, 2010. 
22. Turner G and Hancock RL: Histone methylase activity of adult, embryonic and neoplastic liver tissues. Life Sci II 9: 917-922, 1970.

23. Ballestar E and Esteller M: The epigenetic breakdown of cancer cells: From DNA methylation to histone modifications. Prog Mol Subcell Biol 38: 169-181, 2005.

24. Sakamoto R, Nitta T, Kamikawa Y, Sugihara K, Hasui K, Tsuyama $S$ and Murata F: The assessment of cell proliferation during 9,10-dimethyl-1,2-benzanthracene-induced hamster tongue carcinogenesis by means of histone $\mathrm{H} 3$ mRNA in situ hybridization. Med Electron Microsc 37: 52-61, 2004.

25. Piscopo M, Campisi G, Colella G, Bilancione M, Caccamo S Di Liberto C, Tartaro GP, Giovannelli L, Pulcrano G and Fucci L: $\mathrm{H} 3$ and H3.3 histone mRNA amounts and ratio in oral squamous cell carcinoma and leukoplakia. Oral Dis 12: 130-136, 2006.

26. Sadikovic B, Yoshimoto M, Chilton-MacNeill S, Thorner P, Squire JA and Zielenska M: Identification of interactive networks of gene expression associated with osteosarcoma oncogenesis by integrated molecular profiling. Hum Mol Genet 18: 1962-1975, 2009.

27. Pérez-Magán E, Rodríguez de Lope A, Ribalta T, Ruano Y, Campos-Martín Y, Pérez-Bautista G, García JF, García-Claver A, Fiaño C, Hernández-Moneo JL, et al: Differential expression profiling analyses identifies downregulation of $1 \mathrm{p}, 6 \mathrm{q}$, and $14 \mathrm{q}$ genes and overexpression of $6 p$ histone cluster 1 genes as markers of recurrence in meningiomas. Neuro Oncol 12: 1278-1290, 2010.
28. Sen GL and Blau HM: A brief history of RNAi: The silence of the genes. FASEB J 20: 1293-1299, 2006.

29. Kong Q: RNAi: A novel strategy for the treatment of prion diseases. J Clin Invest 116: 3101-3103, 2006.

30. Wu Z, Li G, Wu L, Weng D, Li X and Yao K: Cripto-1 overexpression is involved in the tumorigenesis of nasopharyngeal carcinoma. BMC Cancer 9: 315, 2009.

31. Sumimoto H, Hirata K, Yamagata S, Miyoshi H, Miyagishi M, Taira K and Kawakami Y: Effective inhibition of cell growth and invasion of melanoma by combined suppression of BRAF (V599E) and Skp2 with lentiviral RNAi. Int J Cancer 118: 472-476, 2006.

32. Vousden KH and Prives C: Blinded by the light: The growing complexity of p53. Cell 137: 413-431, 2009.

33. Levine AJ and Oren M: The first 30 years of p53: Growing ever more complex. Nat Rev Cancer 9: 749-758, 2009. 